\title{
COMPLEX ANALYTIC REALIZATION OF LINKS
}

\author{
WALTER D. NEUMANN AND ANNE PICHON
}

\begin{abstract}
We present the complex analytic and principal complex analytic realizability of a link in a 3-manifold $M$ as a tool for understanding the complex structures on the cone $C(M)$.
\end{abstract}

\section{INTRODUCTION}

Let $(Z, p)$ be a normal complex surface singularity, and $M_{Z}$ its link, i.e., the 3 -manifold obtained as the boundary of a small regular neighbourhood of $p$ in $Z$. Then, $Z$ is locally homeomorphic to the cone $C\left(M_{Z}\right)$ on $M_{Z}$.

Given a surface singularity link $M$, there may exists many different analytical structures on the cone $C(M)$, i.e., normal surfaces singularities $(Z, p)$ whose $M_{Z}$ is homeomorphic to $M$. A natural problem is to understand these analytic structures on $C(M)$. In this paper we present an approach by studying the principal analytic link-theory on $M$. Our aim is to present this point of view to encourage people to pursue this area.

If $C$ is an analytic curve on $Z$ through $p$, set $L_{C}=C \cap M_{Z}$; the pair $\left(M_{Z}, L_{C}\right)$, defined up to diffeomorphism, is the link of $C$. Notice that $L_{C}$ is a link in $M_{Z}$ in the usual topological sense: a disjoint union of circles embedded in a 3 manifold. In this situation we say $(M, L)=\left(M_{Z}, L_{C}\right)$ is analytically realized by $(Z, C)$ or simply analytic. We say that $(M, L)$ is principal analytic if it is analytically realized by a pair $(Z, C)$ such that $C=f^{-1}(0)$, where $f:(Z, p) \rightarrow(\mathbb{C}, 0)$ is a germ of holomorphic function. In other words, the ideal of $\mathcal{O}_{Z, p}$ defining $C$ is principal.

Given a 3-manifold $M$, we then want to

- describe the links $L \subset M$ which are analytic (resp. principal analytic),

- describe how these links distribute among the different analytic structures on $C(M)$.

When $M$ is the 3 sphere, then $Z$ is smooth $[8$, and one deals with plane curves. The two notions, analytic link and principal analytic link, then coincide under the classical name "algebraic link." Their classification is one of the main results of the classical theory of plane curves singularities: such a link is built by repeated cabling operations, and a link $L \subset S^{3}$ is algebraic iff it is obtained by successive cabling operations which satisfy the so-called Puiseux inequalities. For details see [3].

For general $M$ the analytic link theory is still well understood, as a consequence of Grauert [4. The main fact is that analytic realizability is a topological property, so analytic link-theory is no help to understand the analytic structures on $C(M)$ :

Theorem 1.1. If $L \subset M$ is analytic, it can be realized by a curve $C$ on any normal surface singularity whose link is $M$. Moreover, $(M, L)$ is analytic iff it can be given

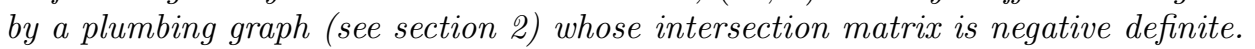


In section 2 we show that the existence of some analytic structure on $C(M)$ for which a link is principal analytic in $M$ is still an easily described topological property (see Thm. 2.1] which treats the more general context of multilinks since the zero-set of a holomorphic function may have multiplicity). But, in contrast to analytic realizability, principal analytic realizability depends on the analytic structure. Here is an explicit example (9, exercise 2.15): the two equations $x^{2}+$ $y^{3}+z^{18}=0$ and $z^{2}+y\left(x^{4}+y^{6}\right)=0$ define two germs $(Z, 0)$ and $\left(Z^{\prime}, 0\right)$ in $\mathbb{C}^{3}$ with homeomorphic links (the plumbing graph $\Delta$ is a string o- $\mathrm{O}-\mathrm{O}$ of 3 vertices with (genus, Euler number) weights $(1,-1),(0,-2)$ and $(0,-2))$. But the link of the holomorphic function $f=z:(Z, 0) \rightarrow(\mathbb{C}, 0)$ does not have a principal realization in $\left(Z^{\prime}, 0\right)$. Indeed, let $\pi: X \rightarrow Z$ and $\pi^{\prime}: X^{\prime} \rightarrow Z^{\prime}$ be two resolutions of $Z$ and $Z^{\prime}$ with dual graph $\Delta$; the compact part of the total transform $(f \circ \pi)$ is $E_{1}+E_{2}+E_{3}$ whereas the maximal cycle on $Z^{\prime}$, realized by the generic hyperplane section, is $2 E_{1}^{\prime}+2 E_{2}^{\prime}+2 E_{3}^{\prime}$, which is greater.

In section 3 we give some results and conjectures about the division of principal analytic links in $M$ among the different analytic structures of $C(M)$. In section 4 we describe the implications for splice singularities, a class of singularities characterized by their principal analytic link theory.

\section{A PRINCIPAL ANALYTIC REALIZATION THEOREM}

We first recall the plumbing representation of the pair $\left(M_{Z}, L_{C}\right)$.

Let $\pi: X \rightarrow Z$ be a resolution of the normal surface germ $(Z, p)$ such that $\pi^{-1}(p)$ is a normal crossing divisor with irreducible components $E_{1}, \ldots, E_{n}$. Denote by $\Delta$ the dual graph associated with $\pi^{-1}(p)$, with vertex $v_{i}$ weighted $\left(g_{i}, e_{i}\right)$ by genus $g_{i}$ and self-intersection $e_{i}=E_{i}^{2}<0$ of the corresponding $E_{i}$ in $X$. Then $M_{Z}$ is homeomorphic to the boundary $M$ of the 4-dimensional manifold obtained from $\Delta$ by the classical plumbing process described in [5]. We call $M$ a plumbing manifold.

By Grauert 4, a plumbing manifold $M$ is the link of a normal complex surface singularity iff it can be given by a plumbing graph $\Delta$ whose associated intersection matrix $I(\Delta)=\left(E_{i} \cdot E_{j}\right)_{1 \leq i, j \leq n}$ is negative definite.

If $C$ is a curve on $Z$, and $\pi: X \rightarrow Z$ a resolution of $X$ such that $\pi^{-1}(C)$ is a normal crossing divisor, let $\Delta$ be the dual graph of the divisor $\pi^{-1}(p)$ decorated with arrows corresponding to the components of the strict transform of $C$ by $\pi$. Then the plumbing graph $\Delta$ completely describes the homeomorphism class of the so-called plumbing link $\left(M_{Z}, L_{C}\right)$.

Suppose now that the curve $C$ is the zero locus of a holomorphic function $f:(Z, p) \rightarrow(\mathbb{C}, 0)$. Let $C_{1}, \ldots C_{r}$ be the irreducible components of $C$ and let $L_{C}=K_{1} \cup \ldots \cup K_{r}$ be its link. Recall that a multilink is a link whose components are weighted by integers. One defines the multilink of $f$ by $L_{f}=m_{1} K_{1} \cup \ldots \cup m_{r} K_{r}$, where $m_{j}$ is the multiplicity of $f$ along the branch $C_{j}$. The plumbing graph $\Delta$ of $\left(M_{Z}, L_{C}\right)$ is then completed by weighting each arrow by the corresponding multiplicity $m_{j}$.

Let $(M, L)$ be a plumbing multilink with graph $\Delta$. For each vertex $v_{i}$ of $\Delta$, let $b_{i}$ be the sum of the multiplicities carried by the arrows stemming from vertex $v_{i}$, and set $b(\Delta)=\left(b_{1}, \ldots, b_{n}\right) \in \mathbb{N}^{n}$. The monodromical system of $\Delta$ is the linear system $I(\Delta)^{t}\left(l_{1}, \ldots, l_{n}\right)+{ }^{t} b(\Delta)=0$ with unknowns $\left(l_{1}, \ldots, l_{n}\right)$, where ${ }^{t}$ means the transposition 
Theorem 2.1. Let $L=m_{1} K_{1} \cup \ldots \cup m_{r} K_{r}$ be a multilink in a 3-manifold $M$ with positive multiplicities $m_{i}$. The following are equivalent:

(i): The multilink $(M, L)$ is principal analytically realized from some analytic structure $(Z, p)$ on $C(M)$.

(ii): $(M, L)$ is a plumbing multilink admitting a plumbing graph $\Delta$ whose monodromical system admits a solution $\left(l_{1}, \ldots, l_{n}\right) \in\left(\mathbb{N}_{>0}\right)^{n}$.

(iii): $(M, L)$ is analytic and $\left[m_{1} K_{1} \cup \ldots \cup m_{r} K_{r}\right]=0$ in $H_{1}(M, \mathbb{Z})$

(iv): $(M, L)$ is a fibered multilink and some power of the monodromy $\Phi: F \rightarrow$ $F$ of the fibration is a product of Dehn twists on a collection of disjoint closed curves which includes all boundary curves.

Proof. The equivalence between (ii), (iii) and (iv) appears in 3 when $M$ is a $\mathbb{Z}$ homology sphere and in 16 (5.4) when $L$ is a link. The methods generalize.

If $f:(Z, p) \rightarrow(\mathbb{C}, 0)$ is a holomorphic function, then a monodromical system is nothing but the well known system in complex geometry $([6], 2.6):(\forall i=$ $\left.1, \ldots, n,(f) \cdot E_{i}=0\right)$, where $(f)$ is the total transform of $f$ in a resolution of $Z$ and $f$ with exceptional divisor $E=\sum_{i=1}^{n} E_{i}$. Then (i) $\Rightarrow$ (ii) is done.

(ii) $\Rightarrow$ (i) is proved in [16] when $L$ is a link (5.5). Let generalize the proof to multilinks. The idea is to perform a surgery along the multilink $L$ in order to realize the new 3-manifold as the boundary of a degenerating families of curves, using a realization theorem of Winters $(\underline{18})$.

Let us consider the plumbing graph $\Delta^{\prime}$ obtained from $\Delta$ by replacing each arrow $v_{i} \mathrm{O} \longrightarrow v_{j}$ by a string of vertices $v_{i} \mathrm{O}-\mathrm{O}-\mathrm{O}-\cdots-\mathrm{O}-\mathrm{O}$ as follows: $v_{i}$ is the vertex carrying the arrow $v_{j}$; set $p_{1}=l_{i}, d_{1}=m_{j}$, and consider the integers $q_{1} \geq 1$ and $r_{1}$ such that $p_{1}=q_{1} d_{1}-r_{1}$, with $0 \leq r_{1}<d_{1}$. Set $p_{2}=d_{1}$ and $d_{2}=r_{1}$, and repeat the process on $p_{2}$ and $d_{2}$ by taking $q_{2} \geq 1$ and $r_{2}$ such that $p_{2}=q_{2} d_{2}-r_{2}$, with $0 \leq r_{2}<d_{1}$. Then iterate the process until $r_{m}=0$. The string has $m$ vertices weighted from $v_{i}$ by the Euler classes $-q_{1},-q_{2}, \ldots,-q_{m}$ and by genus zero.

The monodromical system of $\Delta^{\prime}$ (which has $b\left(\Delta^{\prime}\right)=0$ ) has the following $\left(l_{k}^{\prime}\right)$ as a solution: $l_{k}^{\prime}=l_{k}$ when $v_{k}$ is a vertex of the subgraph $\Delta$, and $l_{k}^{\prime}=d_{k}$ for the vertex $v_{k}$ of the string carrying $-q_{k}$. According to [18, there then exists a degenerating family of curves (i.e., a proper holomorphic family which has no critical value except 0) $g: \Sigma \rightarrow\{z \in \mathbb{C} /|z|<1\}$, whose special fiber $f^{-1}(0)$ has $\Delta^{\prime}$ as dual graph [18.

By Zariski's lemma (e.g., [8]), condition (ii) implies that the intersection matrix $I(\Delta)$ is negative definite. One then obtains from $\Sigma$ a normal surface $Z$ by shrinking to a single point $p$ all the irreducible components of $f^{-1}(0)$ corresponding to vertices of $\Delta$. Then $g$ induces a holomorphic function $Z \backslash\{p\} \rightarrow \mathbb{C}$, which extends by $p \mapsto 0$ to a holomorphic function $f: Z \rightarrow \mathbb{C}$ as $(Z, p)$ is normal. This $f$ realizes $(M, L)$.

\section{DePEndEnCE ON ANALYTIC STRUCtURE}

There exist some 3-manifolds $M$ whose principal analytic knot-theory does not depends on the analytic structure. For example, when $M$ is the link of a rational singularity, then any principal analytic realizable multilink $(M, L)$ is so realizable in any analytic structure $(Z, p)$ on $C(M)$ [1]. The same conclusion holds when $M$ is the link of a minimally elliptic singularity and $L$ is a knot, possibly with multiplicity (17, lemma p. 102). It seems likely that in most, if not all, other cases the principle analytic knot theory is sensitive to analytic structure. We are 
willing to dare a conjecture in the $\mathbb{Z}$-homology sphere case. In this case there is no homological obstruction to the principal analytic realizability (Theorem 2.1 (iii)).

Denote the Brieskorn singularity $\left\{\left(x_{1}, x_{2}, x_{3}\right) \in \mathbb{C}^{3} \mid x_{1}^{p}+x_{2}^{q}+x_{3}^{r}=0\right\}$ by $V(p, q, r)$ and its link by $M(p, q, r)$. If $p, q, r$ are pairwise coprime then $M=$ $M(p, q, r)$ is a $\mathbb{Z}$-homology sphere. The only $\mathbb{Z}$-homology sphere links of rational and minimally elliptic singularities are $M(2,3,5)$ (rational) and $M(2,3,7)$ and $M(2,3,11)$ (minimally elliptic). So the principal knot theory of these is completely understood (even the principal analytic link theory for $M(2,3,5)$ ).

Conjecture 3.1. Let $M$ be a $\mathbb{Z}$-homology sphere link other than $M(2,3,5), M(2,3,7)$, $M(2,3,11)$. Then for any analytic structure $(Z, p)$ on $C(M)$ there exists an analytic knot in $M$ which is not realized by a holomorphic germ $(Z, p) \rightarrow(\mathbb{C}, 0)$.

We can prove the conjecture in many cases. We give two examples to illustrate the arguments. $M(p, q, r)$ is Seifert fibered with singular fibers $L_{1}, L_{2}, L_{3}$ realized as principal analytic knots in $V(p, q, r)$ by $L_{i}=M \cap\left\{x_{i}=0\right\}$.

1) In $M(2,3,13)$ let $L$ be the $(2,1)$-cable on $L_{3}$. As $L$ satisfies condition (iii) of Theorem 2.1 it is principal analytic in some analytic structure.

2) In $M(3,4,19)$ let $L$ be the $(2,3)$-cable on $L_{3}$. As $L$ satisfies condition (iii) of Theorem 2.1 it is principal analytic in some analytic structure.

Proposition 3.1. (1) Let $(Z, p)$ be an analytic structure on $C(M(2,3,13))$ such that $L_{3}$ is realized by a holomorphic function $f_{3}:(Z, p) \rightarrow(\mathbb{C}, 0)$. Then $L$ is not realized by any $f:(Z, p) \rightarrow(\mathbb{C}, 0)$ on $(Z, p)$.

(2) Let $(Z, p)$ be an analytic structure on $C(M(3,4,19))$ such that both $L_{2}$ and $L_{3}$ are realized on $(Z, p)$ by $f_{2}, f_{3}:(Z, p) \rightarrow(\mathbb{C}, 0)$ respectively. Then, $L$ is not realized by any $f:(Z, p) \rightarrow(\mathbb{C}, 0)$.

Proof. (1) Assume the contrary. Let $E_{i}, i=1, \ldots, 5$ be the irreducible components of the exceptional divisor of the minimal resolution $\pi: \Sigma \rightarrow Z$ of $(Z, p)$ as in the figure below.

The total transform of $f_{3}$ is $\left(f_{3} \circ \pi\right)=3 E_{1}+2 E_{2}+6 E_{3}+E_{4}+E_{5}+l_{3}$, so its multiplicity on $E_{4}$ is 1 , which means that $f_{3}$ is a local coordinate on the transverse curve $f^{-1}(0)$ to $E_{4}$, so $f^{-1}(0)$ is smooth. Therefore, the Milnor fibre $F_{t}$ of $f$, $t \neq 0$, is a disk, as it is a smoothing of $f^{-1}(0)$. But the total transform of $f$ is $(f \circ \pi)=6 E_{1}+4 E_{2}+12 E_{3}+2 E_{4}+E_{5}+l$, which leads to $\chi\left(F_{t}\right)=6+4-12-2+1=-3$. Contradiction.
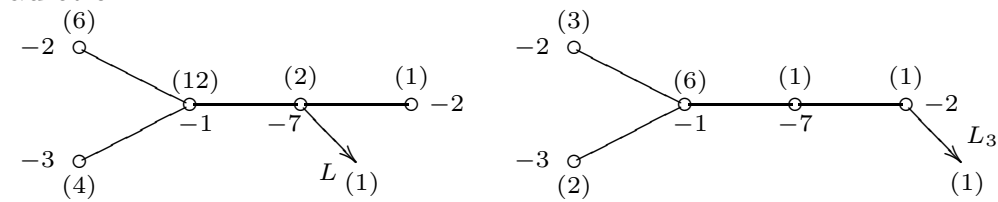

2) Assume the contrary to the proposition. Let $f:(Z, p) \rightarrow(\mathbb{C}, 0)$ be such that $L$ is the link of $f^{-1}(0)$. The splice diagram is as follows:

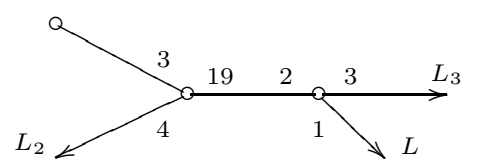

The semi-group of values $\Gamma(C)$ of the curve $C=f^{-1}(0)$ contains the two values 2 and 9 associated with the functions $f_{2}$ and $f_{3}$ (these are computed as the product of 
splice diagram weights adjacent to the path between the corresponding arrowheads). So $\Gamma(C)$ contains the semi-group $\langle 2,9\rangle=\left\{2,4,6,8,8+\mathbb{Z}^{+} \ldots\right\}$ which has 4 missing numbers $(1,3,5$ and 7$)$. Therefore, the $\delta$-invariant $\delta(C)$ of $C$, which counts the number of gaps in $\Gamma(C)$, is at most 4 , so $\mu(C)=2 \delta \leq 8$. But the multiplicities of $C$ leads to $\chi\left(F_{t}\right)=12+9-36-6+2=-19$. Then $\mu=20$. Contradiction.

In view of the above discussion, the following question is natural.

Question 3.1. Let $M$ be a surface singularity link. Do there exist analytic links in $M$ that have the ubiquity property, i.e., that are principal analytic in any analytic structure $(Z, p)$ with link $M$ ?

A positive answer is given by the following theorem, which is a slight improvement of Theorem 4.1 of [2]:

Theorem 3.1. Let $g_{i}$ be the genus weights of a negative definite plumbing for $M$ and $L$ be the $\left(\sum\left(2 g_{i}+1\right)\right)$-component link consisting of the boundaries of $2 g_{i}+1$ transverse disks to each base curve $E_{i}$ of the plumbing. Then $L$ is principal analytic in any analytic structure $(Z, 0)$ on $C(M)$.

If $M$ is a $\mathbb{Q}$-homology sphere this ubiquitous link has one component for each vertex of the negative definite plumbing graph (since each $g_{i}$ is 0 ). It seems unlikely that this can be reduced much in general, although in the rational and minimally elliptic cases 1 component suffices.

In the original version of this theorem in 2], $\mathrm{L}$ had one extra component for each edge of the plumbing graph. The proof of the above improvement is a small modification of that proof, but the 8 page limit on contributions to this volume leaves insufficient space to give it here.

\section{Singularities of SPlice type}

In [12, 13 the first author and J. Wahl introduce an important class of singularities with $\mathbb{Q}$-homology sphere links called "splice-quotient singularities," or briefly "splice singularities." This class includes all rational singularities and all minimal elliptic singularities with $\mathbb{Q}$-homology sphere links. This was first proved by Okuma [15], see also the appendix to [12. It now follows from the following characterization of splice singularities by their principal analytic knot theory. We assume $M$ is a $\mathbb{Q}-$-homology sphere. The dual resolution graph is then a tree.

Theorem 4.1 (End Curve Theorem (14). To each leaf of the dual resolution graph there is associated a knot in $M$, namely the link of a transverse curve to the corresponding exceptional curve of the resolution; the singularity is splice if and only if each of these knots, taken with some multiplicity, has a principal analytic realization.

The property of being rational or minimally elliptic is topologically determined, so any singularity with one of these topologies is splice. But in general the same topology may support both splice and non-splice singularities. For example, Proposition 3.1 implies for either of the examples it addresses, that if one takes an analytic structure for which the knot $L$ is principal, then that singularity is not splice.

Associated to any plumbed $\mathbb{Q}$-homology sphere is a simplified version of the resolution graph, called the splice diagram. A fundamental property of the splice diagrams of links of splice singularities is the so-called "semigroup condition" (loc. 
cit.). The following question is of fundamental importance, since a $\mathbb{Z}$-sphere counterexample would give a complete intersection singularity with $\mathbb{Z}$-homology sphere link that is not splice (conjectured not to exist), and would give a likely candidate also to contradict the Casson Invariant Conjecture of [11.

Question 4.1. Is the principal knot theory of a splice singularity topologically determined? Specifically, is a knot $L \subset M$ with multiplicity principal analytic if and only if it represents zero in homology of $M$ and the splice diagram for $(M, L)$ satisfies the semigroup condition?

Acknowledgments: The first author acknowledges the support of the NSF and the NSA for this research.

\section{REFERENCES}

[1] M. Artin, On isolated rational singularities of surfaces, Amer. J. Math. 88 (1966), 129-136.

[2] C. Caubel, A. Nemethi, P. Popescu-Pampu, Milnor open books and Milnor Fillable Contact 3-manifolds, arXiv math.SG/0409160

[3] D. Eisenbud, W. D. Neumann, Three dimensional link theory and invariants of plane curves singularities, Annals of Mathematics Studies 110, Princeton University Press, 1985.

[4] H. Grauert, Über Modifikation und Exceptionelle analytische Mengen, Math. Ann. 146 (1962), 331-368

[5] F. Hirzebruch, W. D. Neumann, S. S. Koh, Differentiable manifolds and quadratic forms, Dekker Publ., 1971.

[6] H. B. Laufer, Normal two-dimensional singularities, Ann. of Math. Studies, No. 71. Princeton University Press, Princeton, 1971.

[7] H. B. Laufer, On minimally elliptic singularities, Amer. J. Math. 99 (1977), 1257-1295.

[8] D. Mumford, The topology of normal singularities of an algebraic surface and a criterion for simplicity, Inst. Hautes Études Sci., Publ. Math. 9 (1961), 1-87.

[9] A. Nemethi, Five lectures on normal surface singularities, Bolyai Soc. Math. Stud., 8, Low dimensional topology (Eger, 1996/Budapest, 1998), 269-351.

[10] W.D. Neumann, A calculus for plumbing applied to the topology of complex surface singularities and degenerating complex curves, Trans. Amer. Math. Soc. 268 (1981), 299-343.

[11] W.D. Neumann, J. Wahl, Casson invariant of links of singularities, Comment. Math. Helv. 65 (1990), 58-78.

[12] W.D. Neumann and J. Wahl, Complete intersection singularities of splice type as universal abelian covers, Geom. Topol. 9 (2005), 699-755.

[13] W.D. Neumann and J. Wahl, Complex surface singularities with homology sphere links, Geom. Topol. 9 (2005), 757-811.

[14] W.D. Neumann and J. Wahl, In preparation.

[15] T. Okuma, Universal abelian covers of certain surface singularities, arXiv math.AG/0503733

[16] A. Pichon, Fibrations sur le cercle et surfaces complexes, Ann. Inst. Fourier 51 (2001), 337374.

[17] M. Reid, Chapters on Algebraic Surfaces, from: "Complex algebraic geometry (Park City, UT, 1993)", IAS/Park City Math. Ser. 3, Amer. Math. Soc. (1997) 3-159.

[18] G. Winters, On the existence of certain families of curves, Amer. J. Math. 96 (1974), 215-228.

Department of Mathematics, Barnard College, Columbia University, New-York, Ny 10027, USA, E-MAIL: NEUMANN@MATH.COLUMBIA.EDU

Institut de Mathématiques de Luminy, UMR 6206 CNRS, Campus de Luminy - Case 907, 13288 Marseille Cedex 9, France, E-mail: Pichon@iml.univ-mrs.Fr 